\title{
Effectiveness of expanding annual mass azithromycin distribution treatment coverage for trachoma in Niger: a cluster randomised trial
}

\author{
Abdou Amza, ${ }^{1}$ Boubacar Kadri, ${ }^{1}$ Beido Nassirou, ${ }^{1}$ Sun Y Cotter, ${ }^{2}$ \\ Nicole E Stoller, ${ }^{2}$ Sheila KWest, ${ }^{3}$ Robin L Bailey, ${ }_{1}^{4}$ Travis C Porco, ${ }^{2,5,6}$ Bruce D Gaynor, ${ }^{2,5}$ \\ Jeremy D Keenan, ${ }^{2,5,6}$ Thomas M Lietman, ${ }^{2,5,6}$ Catherine E Oldenburg ${ }^{2,5,6}$
}

Programme FSS/Université Abdou Moumouni de Niamey, Programme National de Santé Oculaire, Niamey, Niger ${ }^{2}$ F.I. Proctor Foundation, University of California San Francisco, San Francisco, California, USA

${ }^{3}$ Dana Center for Preventive Ophthalmology, Wilmer Eye Institute, Johns Hopkins University, Baltimore, Maryland, USA

${ }^{4}$ Clinical Research Unit, Department of Infectious and Tropical Diseases, London School of Hygiene \& Tropical Medicine, London, UK ${ }^{5}$ Department of Ophthalmology, University of California San Francisco, San Francisco, California, USA

${ }^{6}$ Department of Epidemiology \& Biostatistics, University of California San Francisco, San Francisco, California, USA

\section{Correspondence to}

Dr Catherine E Oldenburg, 513 Parnassus Ave, San Francisco, CA 94143, USA; catherine. oldenburg@ucsf.edu

Received 21 June 2017 Revised 7 August 2017 Accepted 16 August 2017 Published Online First 11 September 2017
Check for updates

To cite: Amza A, Kadri B

Nassirou $B$, et al.

$\mathrm{Br} J$ Ophthalmol

2018;102:680-686.

\begin{abstract}
Background/aims The WHO recommends 3-5 years of annual mass azithromycin distribution with at least $80 \%$ treatment coverage to districts with active trachoma prevalence over 10\% among children. Here, we assess the efficacy of expanding the coverage target to at least $90 \%$ for trachoma control in a mesoendemic region of Niger.
\end{abstract}

Methods Twenty-four communities were randomised to a single day of azithromycin distribution with a coverage target of $80 \%$ of the community or up to 4 days of treatment, aiming for greater than $90 \%$ coverage. Distributions were annual and individuals above 6 months of age were treated. Children under 5 years of age were monitored for ocular chlamydia infection and active trachoma.

Results At baseline, ocular chlamydia prevalence was $20.5 \%(95 \% \mathrm{Cl} 9.8 \%$ to $31.2 \%)$ in the standard coverage arm and $21.9 \%(95 \% \mathrm{Cl} 11.3 \%$ to $32.5 \%)$ in the enhanced coverage arm, which reduced to $4.6 \%$ (95\% Cl $0 \%$ to $9.5 \%, \mathrm{p}=0.008)$ and $7.1 \%(95 \% \mathrm{Cl}$ $2.7 \%$ to $11.4 \%, p<0.001)$ at 36 months, respectively. There was no significant difference in 36-month ocular chlamydia prevalence between the two arms $(p=0.21)$. There was no difference in the rate of decline in ocular chlamydia between the two arms in a repeated measures model $(p=0.80)$.

Conclusions For annual mass azithromycin distribution programme to an entire community, there may be no additional benefit of increasing antibiotic coverage above the WHO's $80 \%$ target.

Trial registration number NCT00792922, postresults.

\section{INTRODUCTION}

The WHO recommends 3-5 years of annual mass azithromycin distribution in districts with active trachoma (trachomatous inflammation-follicular; TF) prevalence above $10 \%$ in children aged $1-9$ years, with treatment coverage of at least $80 \%$. Impact surveys should be conducted afterwards, with possible continuation of mass drug administration depending on the prevalence of active trachoma. ${ }^{1}$ However, despite many years of mass drug administration, trachoma control has been difficult to achieve in some communities under the current guidelines. For example, in communities in Tanzania with 10\%-20\% TF prevalence at baseline,
3 years of annual mass azithromycin distribution with approximately $80 \%$ azithromycin coverage were not sufficient for control of infection. ${ }^{2}$ In Ethiopia, mass azithromycin distribution led to dramatic reduction in ocular chlamydia infection prevalence, but infection rapidly returned when antibiotic distribution was discontinued. ${ }^{3}$

Alternative antibiotic distribution strategies may be required to achieve control of trachoma in communities with residual disease. Increasing antibiotic coverage targets is one option for improving trachoma control with mass azithromycin distributions. ${ }^{4}$ Treating a greater number of individuals within the community may reduce the reservoir of ocular chlamydia in the community and increase the likelihood of achieving herd protection. ${ }^{56}$

The Partnership for the Rapid Elimination of Trachoma (PRET) was a series of three cluster-randomised trials in Tanzania, ${ }^{78}$ The Gambia ${ }^{9}$ and Niger ${ }^{10}$ designed to assess the efficacy of increasing coverage targets from at least $80 \%$ to at least $90 \%$ for control of trachoma. In communities in the Gambia with low baseline TF prevalence (6.5\%) and very little ocular Chlamydia trachomatis infection, there was no difference in communities randomised to a $90 \%$ coverage target compared with $80 \%$; however, there was no $C$. trachomatis infection detected in any communities after a single round of mass azithromycin distribution. ${ }^{9}$ In Tanzania, increasing coverage targets to at least $90 \%$ did not lead to a significant difference in ocular chlamydia prevalence after 36 months. ${ }^{7}$ Here, we report the results of the PRET-Niger trial comparing annual mass azithromycin coverage targets of at least $80 \%$ versus at least $90 \%$.

\section{MATERIALS AND METHODS}

\section{Participants and procedures}

Complete methods for the PRET-Niger trial have been previously reported (ClinicalTrials.gov NCT00792922, post-results). ${ }^{10}$ Participants were enrolled in Matamèye District, Zinder Region, Niger from May 2010 until August 2013. Here, we include 24 grappes (smallest government health unit; henceforth, 'community') from 6 Centres de Santé Intégrées (CSI) that were randomised to annual mass azithromycin distribution with a target of at least $80 \%$ antibiotic coverage ('standard') or annual mass azithromycin distribution with up to 4 days of treatment and a target antibiotic coverage 


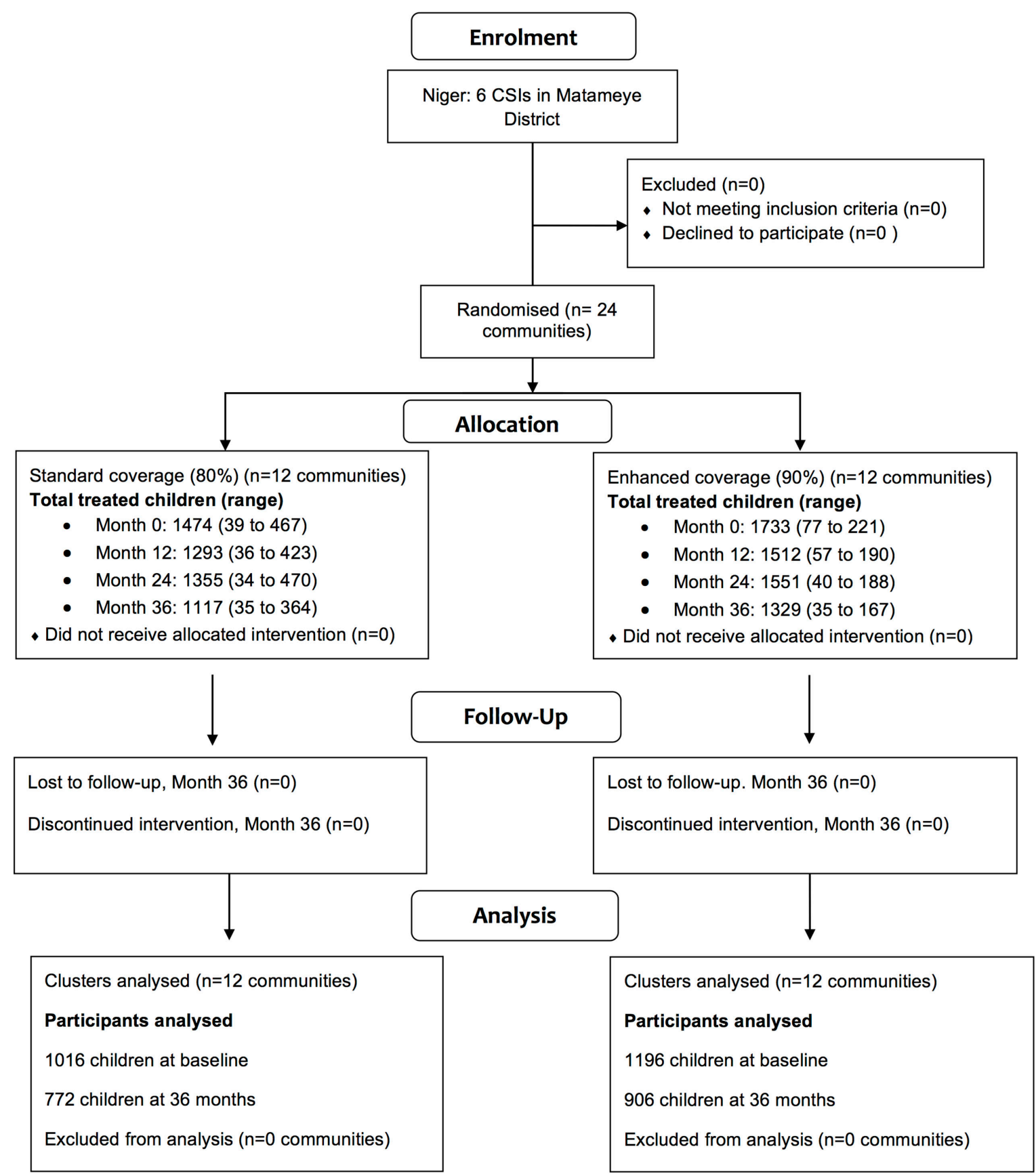

Figure 1 Flow diagram of study communities.

of at least 90\% ('enhanced'). Ethical approval was obtained from the Committee on Human Research at the University of California, San Francisco and the Comité d'Ethique du Niger. The trial was implemented according to the Declaration of Helsinki. We obtained verbal consent from each local community chief prior to randomisation. Each individual or guardian provided verbal informed consent prior to examination due to low literacy rates in the study area.

\section{Eligibility criteria}

Communities were eligible for the study if they had a population between 250 and 600 at the most recent government census and had at least $10 \%$ prevalence of active trachoma (TF and/or trachomatous inflammation-intense (TI)). Baseline trachoma prevalence was measured prior to the first mass antibiotic treatment. A random sample of 100 children aged 0-60 per community was taken from a door-to-door census and was assessed for active trachoma according to the WHO simplified grading system. ${ }^{11} 12$

\section{Randomisation}

Communities were randomised by stratified block randomisation within each CSI by high or low trachoma prevalence in children. Within a given CSI, communities above the median trachoma prevalence were considered to be 'high', and those below the median were considered to be 'low'. The random allocation sequence was generated by TCP using R V.2.12 (R Foundation for Statistical Computing, Vienna, Austria; http:// www.r-project.org). 


\section{Intervention}

All communities received four rounds of annual mass azithromycin distribution (at months 0, 12, 24 and 36). In the standard coverage arm, a single dose of azithromycin, $20 \mathrm{mg} / \mathrm{kg}$ for children and $1 \mathrm{~g}$ for adults, was distributed during a single day, aiming for a coverage target of $80 \%$ or greater of children and adults. In the enhanced coverage arm, communities received up to three follow-up visits to achieve coverage of $90 \%$ or greater of children and adults. Treatment teams visited communities up to four times and discontinued visits once treatment coverage exceeded $90 \%$ or once they completed four treatment visits. Children under 6 months of age and those known to be allergic to macrolides were offered tetracycline ointment $(1 \%)$ to be applied to both eyes two times per day for 6 weeks.

\section{Outcome measurement}

An annual census was conducted in each community. At baseline and months $6,12,18,24,30$ and 36 , a random sample of 100 children aged $0-5$ years per community (or all children if a given community had fewer than 100 children) was selected from the most recent census for examination. Clinical examination for TF and TI was performed according to the WHO simplified grading system by examiners trained and certified by experienced graders. ${ }^{11}$ Ocular chlamydial infection was assessed from a Dacron swab of the everted right upper tarsal conjunctiva, collected without media. Swabs were placed on ice for $<8$ hours while in the field, then stored in a $-20^{\circ} \mathrm{C}$ freezer before being transported at $4^{\circ} \mathrm{C}$ to the University of California, San Francisco, where they were kept at $-80^{\circ} \mathrm{C}$ until processed. Swabs were pooled by community into pools of five plus a remainder pool to save processing costs. The pools were processed with Amplicor PCR testing, and prevalence was estimated from pooled results as previously described. ${ }^{13}$

\section{Sample size}

For the overall trial, we estimated that 48 communities (12 per arm in a $2 \times 2$ factorial design) would yield greater than $80 \%$
Table 1 Baseline characteristics by study arm

\begin{tabular}{lll}
\hline & \multicolumn{2}{l}{ Mean (95\% Cl) } \\
\cline { 2 - 3 } & Standard coverage & Enhanced coverage \\
\hline & $(\mathrm{n}=12)$ & $(\mathrm{n}=12)$ \\
\hline Community residents & $557(261$ to 849$)$ & $525(182$ to 2071$)$ \\
\hline Proportion female & $52.7 \%(51.0 \%$ to & $51.9 \%(50.9 \%$ to \\
& $54.3 \%)$ & $52.8 \%)$ \\
\hline Proportion aged 0-5 years & $29.6 \%(28.0 \%$ to & $27.7 \%(26.0 \%$ to \\
& $31.1 \%)$ & $29.3 \%)$ \\
\hline $\begin{array}{l}\text { Prevalence of ocular chlamydia in } \\
\text { children aged 0-5 years }\end{array}$ & $21.9 \%(11.3 \%$ to & $20.5 \%(9.8 \%$ to \\
Prevalence of trachomatous & $32.5 \%)$ & $31.2 \%)$ \\
inflammation-follicular in children & $28.4 \%(19.6 \%$ to & $27.0 \%(16.0 \%$ to \\
aged 0-5 years & $37.2 \%)$ & $38.0 \%)$ \\
\hline
\end{tabular}

power to detect an absolute difference of $6 \%$ prevalence of ocular chlamydia infection in children, assuming a SD in the community-level prevalence of $5 \%$. The primary analysis for the present report was the difference in prevalence of ocular chlamydia between the enhanced and standard antibiotic coverage arms for the annual mass azithromycin distribution strategy.

\section{Statistical methods}

The prespecified primary analysis used a linear regression model to compare 36-month prevalence of ocular chlamydia infection in children aged $0-5$ years between communities randomised to standard versus enhanced coverage, adjusting for baseline ocular chlamydia prevalence. A square root transformation of ocular chlamydia infection prevalence was used for all analyses, per our prespecified analysis plan. As prespecified secondary analyses, we assessed the reduction in ocular chlamydia prevalence between baseline and 36 months with a paired t-test. We also used a mixed effects model to assess the rate of change in ocular chlamydia prevalence over time at each time point $(0,6$, $12,18,24,30$ and 36 months), with randomisation arm, time

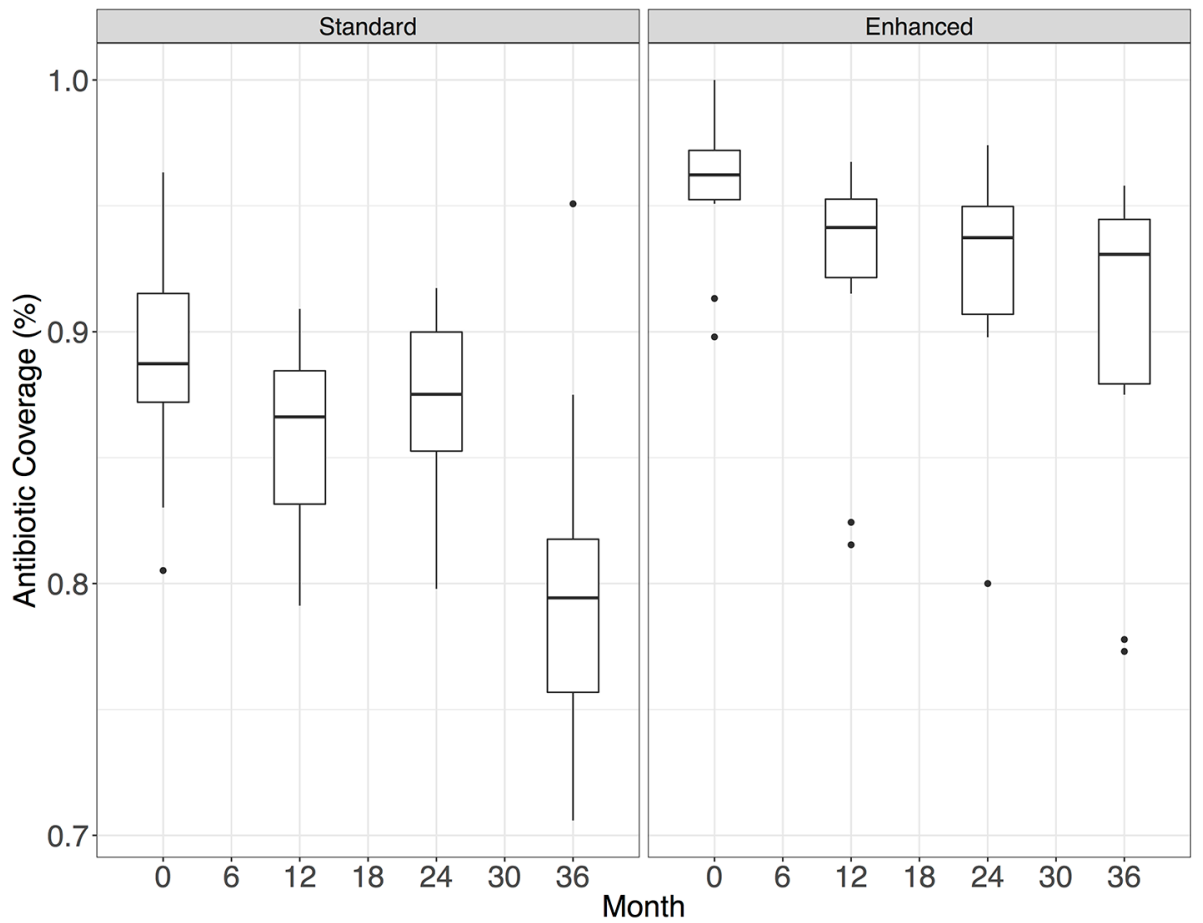

Figure 2 Antibiotic coverage among children aged $0-5$ years during mass antibiotic distributions by study visit. 
Table 2 Longitudinal prevalence of ocular chlamydia among a random sample of 0-5-year-old children

\begin{tabular}{|c|c|c|c|c|c|c|c|c|c|c|c|c|c|c|}
\hline \multirow{2}{*}{ Community } & \multicolumn{14}{|c|}{ Prevalence of ocular chlamydia, $0-5$-year-old children } \\
\hline & \multicolumn{2}{|c|}{ Month 0} & \multicolumn{2}{|c|}{ Month 6} & \multicolumn{2}{|c|}{ Month 12} & \multicolumn{2}{|c|}{ Month 18} & \multicolumn{2}{|c|}{ Month 24} & \multicolumn{2}{|c|}{ Month 30} & \multicolumn{2}{|c|}{ Month 36} \\
\hline \multicolumn{15}{|c|}{ Standard coverage } \\
\hline 1 & $29.7 \%$ & $(30 / 101)$ & $2.9 \%$ & $(3 / 102)$ & $2.9 \%$ & $(3 / 102)$ & $4.9 \%$ & $(5 / 103)$ & $6.1 \%$ & (6/99) & $3.0 \%$ & $(3 / 100)$ & $12.0 \%$ & $(10 / 83)$ \\
\hline 2 & $8.2 \%$ & $(4 / 49)$ & $2.3 \%$ & $(1 / 43)$ & $2.2 \%$ & $(1 / 45)$ & 0 & $(0 / 47)$ & 0 & $(0 / 41)$ & 0 & $(0 / 40)$ & 0 & $(0 / 41)$ \\
\hline 3 & $8.2 \%$ & $(8 / 97)$ & 0 & $(0 / 99)$ & 0 & $(0 / 50)$ & $1.4 \%$ & $(1 / 70)$ & $4.3 \%$ & $(3 / 69)$ & $1.8 \%$ & $(1 / 55)$ & $2.2 \%$ & $(1 / 46)$ \\
\hline 4 & $51.2 \%$ & $(22 / 43)$ & $19.5 \%$ & $(8 / 41)$ & $13.5 \%$ & $(5 / 37)$ & 0 & $(0 / 38)$ & 0 & $(0 / 41)$ & $4.8 \%$ & $(2 / 42)$ & $22.5 \%$ & $(9 / 40)$ \\
\hline 5 & $25.0 \%$ & $(18 / 72)$ & $2.9 \%$ & $(2 / 69)$ & $2.7 \%$ & $(2 / 73)$ & $1.5 \%$ & $(1 / 67)$ & $2.7 \%$ & $(2 / 74)$ & 0 & $(0 / 81)$ & 0 & $(0 / 70)$ \\
\hline 6 & $12.8 \%$ & $(14 / 109)$ & $4.0 \%$ & $(4 / 101)$ & $2.0 \%$ & (2/99) & 0 & $(0 / 105)$ & 0 & $(0 / 100)$ & 0 & $(0 / 101)$ & 0 & $(0 / 74)$ \\
\hline 7 & $1.6 \%$ & $(1 / 64)$ & 0 & $(0 / 64)$ & 0 & $(0 / 56)$ & 0 & $(0 / 64)$ & 0 & (0/58) & 0 & $(0 / 67)$ & 0 & $(0 / 44)$ \\
\hline 8 & $20.0 \%$ & $(10 / 50)$ & 0 & $(0 / 48)$ & $2.6 \%$ & $(1 / 38)$ & $2.6 \%$ & $(1 / 39)$ & $2.7 \%$ & $(1 / 37)$ & 0 & $(0 / 34)$ & 0 & $(0 / 38)$ \\
\hline 9 & $3.0 \%$ & $(3 / 100)$ & $1.0 \%$ & $(1 / 104)$ & $1.0 \%$ & $(1 / 102)$ & $1.3 \%$ & $(1 / 79)$ & $1.0 \%$ & $(1 / 101)$ & 0 & $(0 / 95)$ & 0 & $(0 / 91)$ \\
\hline 10 & $30.6 \%$ & (60/196) & $3.1 \%$ & (6/194) & $4.4 \%$ & $(8 / 181)$ & $2.7 \%$ & $(5 / 186)$ & $2.5 \%$ & $(4 / 163)$ & 0 & $(0 / 170)$ & $1.5 \%$ & $(2 / 132)$ \\
\hline 11 & $7.4 \%$ & $(4 / 54)$ & $3.6 \%$ & $(2 / 55)$ & $6.3 \%$ & $(3 / 48)$ & $4.0 \%$ & $(2 / 50)$ & $2.4 \%$ & $(1 / 42)$ & 0 & $(0 / 43)$ & 0 & $(0 / 46)$ \\
\hline 12 & $48.1 \%$ & $(39 / 81)$ & 0 & $(0 / 83)$ & 0 & $(0 / 77)$ & $9.2 \%$ & (7/76) & $24.7 \%$ & $(19 / 77)$ & $8.2 \%$ & $(7 / 85)$ & $16.4 \%$ & $(11 / 67)$ \\
\hline \multicolumn{15}{|c|}{ Enhanced coverage } \\
\hline 13 & $3.1 \%$ & $(3 / 97)$ & $1.0 \%$ & $(1 / 103)$ & $2.9 \%$ & $(3 / 102)$ & 0 & $(0 / 108)$ & $1.3 \%$ & $(1 / 76)$ & $2.6 \%$ & $(1 / 39)$ & $4.8 \%$ & $(1 / 21)$ \\
\hline 14 & $39.2 \%$ & $(40 / 102)$ & $11.2 \%$ & $(12 / 107)$ & $13.3 \%$ & $(14 / 105)$ & $2.9 \%$ & $(3 / 103)$ & $5.4 \%$ & $(6 / 112)$ & $4.7 \%$ & $(4 / 85)$ & $8.4 \%$ & $(9 / 107)$ \\
\hline 15 & $31.4 \%$ & $(32 / 102)$ & $1.9 \%$ & $(2 / 106)$ & $5.8 \%$ & $(6 / 104)$ & $27.9 \%$ & $(29 / 104)$ & $28.2 \%$ & $(20 / 103)$ & $18.5 \%$ & $(20 / 108)$ & $11.5 \%$ & $(11 / 96)$ \\
\hline 16 & $34.6 \%$ & $(37 / 107)$ & $8.8 \%$ & $(9 / 102)$ & $19.8 \%$ & $(20 / 101)$ & $14.0 \%$ & $(14 / 100)$ & $15.2 \%$ & $(16 / 105)$ & $17.6 \%$ & $(18 / 102)$ & $21.7 \%$ & $(18 / 83)$ \\
\hline 17 & $9.3 \%$ & $(7 / 75)$ & $4.8 \%$ & $(3 / 63)$ & $4.1 \%$ & $(2 / 49)$ & $6.5 \%$ & $(3 / 46)$ & $13.2 \%$ & $(5 / 38)$ & $5.9 \%$ & $(2 / 34)$ & $12.5 \%$ & $(1 / 8)$ \\
\hline 18 & $58.0 \%$ & $(58 / 100)$ & $8.7 \%$ & $(9 / 104)$ & $10.9 \%$ & $(11 / 101)$ & $1.0 \%$ & $(1 / 102)$ & $2.0 \%$ & $(2 / 101)$ & 0 & $(0 / 104)$ & 0 & $(0 / 89)$ \\
\hline 19 & $25.0 \%$ & $(31 / 124)$ & $10.7 \%$ & $(12 / 112)$ & $8.2 \%$ & (8/98) & $1.1 \%$ & $(1 / 94)$ & $5.5 \%$ & $(5 / 91)$ & 0 & $(0 / 92)$ & 0 & $(0 / 84)$ \\
\hline 20 & $23.7 \%$ & $(27 / 114)$ & $5.8 \%$ & $(6 / 103)$ & $15.7 \%$ & $(16 / 102)$ & $10.5 \%$ & $(11 / 105)$ & $17.6 \%$ & $(18 / 102)$ & $7.8 \%$ & $(8 / 103)$ & $5.5 \%$ & $(6 / 110)$ \\
\hline 21 & $10.9 \%$ & $(11 / 101)$ & $2.0 \%$ & (3/107) & $2.2 \%$ & $(2 / 92)$ & 0 & $(0 / 109)$ & 0 & $(0 / 100)$ & 0 & $(0 / 90)$ & 0 & $(0 / 79)$ \\
\hline 22 & $17.0 \%$ & $(18 / 106)$ & $2.8 \%$ & $(3 / 107)$ & $0.9 \%$ & $(1 / 107)$ & $0.9 \%$ & $(1 / 109)$ & $4.7 \%$ & $(5 / 106)$ & $10.1 \%$ & $(12 / 119)$ & $13.5 \%$ & $(14 / 104)$ \\
\hline 23 & $4.9 \%$ & $(5 / 103)$ & $2.0 \%$ & $(2 / 101)$ & $1.0 \%$ & $(1 / 103)$ & $1.0 \%$ & $(1 / 101)$ & 0 & $(0 / 85)$ & $1.1 \%$ & $(1 / 92)$ & 0 & $(0 / 83)$ \\
\hline 24 & $6.2 \%$ & $(4 / 65)$ & $14.3 \%$ & $(10 / 70)$ & $3.8 \%$ & (2/52) & $6.9 \%$ & $(4 / 58)$ & $1.9 \%$ & $(1 / 52)$ & $1.7 \%$ & $(1 / 60)$ & $7.1 \%$ & $(3 / 42)$ \\
\hline
\end{tabular}

point and a time point by randomisation arm interaction term as fixed effects and community as a random effect. We used an identical analytic strategy for clinical trachoma outcomes. All $\mathrm{p}$ values were calculated using an exact permutation test. All analyses were intention-to-treat, used a two-sided test and were conducted in R V.3.3.1 (R Foundation for Statistical Computing) at the community level.

\section{RESULTS}

Twenty-four communities with 12991 individuals were randomised to either standard or enhanced azithromycin coverage (figure 1). Communities randomised to standard azithromycin coverage had a mean of 141 (range 44-580) children aged 0-5 years, compared with 151 (range 78-242) in the enhanced coverage arm. Table 1 displays baseline characteristics of communities included in the study. Figure 2 displays antibiotic coverage among children aged $6-59$ months by arm at each time point. Antibiotic coverage was generally above $90 \%$ for communities in the enhanced arm and below $90 \%$ for communities in the standard coverage arm.

Table 2 displays ocular chlamydia prevalence in each study village by time point. At baseline, ocular chlamydia prevalence was $21.9 \%$ (95\% CI $11.3 \%$ to $32.5 \%$ ) in the enhanced coverage arm and $20.5 \%$ (95\% CI 9.8\% to $31.2 \%$ ) in the standard coverage arm. By 36 months, ocular chlamydia prevalence reduced to $7.1 \%(95 \%$ CI $2.7 \%$ to $11.4 \%, \mathrm{p}<0.001)$ in the enhanced communities and $4.6 \%$ (95\% CI $0 \%$ to $9.5 \%, \mathrm{p}=0.008)$ in the standard coverage arm. In a model with terms for study arm and baseline ocular chlamydia prevalence, there was no difference in 36-month ocular chlamydia prevalence between the two arms (mean adjusted difference $2.2 \%, 95 \%$ CI to $-3.0 \%$ to $7.4 \%$, $\mathrm{p}=0.21$ ). In a repeated measures model, there was no difference in rate of decline in ocular chlamydia prevalence between the two arms $(\mathrm{p}=0.80$ for interaction of arm by time; figure 3$)$.

At baseline, TF prevalence was $28.4 \%$ (95\% CI $20.0 \%$ to $37.2 \%)$ in the enhanced coverage arm and $27.0 \%(95 \% \mathrm{CI}$ $16.0 \%$ to $38.0 \%$ ) in the standard coverage arm. TF prevalence reduced to $8.9 \%(95 \% \mathrm{CI} 3.3 \%$ to $14.4 \%, \mathrm{p}<0.001)$ and $7.1 \%$ $(95 \%$ CI $2.1 \%$ to $12.0 \%, \mathrm{p}<0.001)$ in the enhanced and standard coverage arms, respectively. At 36 months, there was no significant difference in TF prevalence between the enhanced and standard arms (mean adjusted difference 1.4\%, 95\% CI to $-4.1 \%$ to $6.8 \%, p=0.94)$. There was no difference in change over time in TF prevalence between the enhanced and standard arms ( $p=0.36$ for interaction of arm by time; figure 4$)$.

\section{DISCUSSION}

Consistent with studies from The Gambia ${ }^{9}$ and Tanzania, ${ }^{14}$ we found no difference in ocular chlamydia or TF prevalence in communities randomised to enhanced azithromycin coverage compared with the standard WHO target. These studies were conducted in hypoendemic (The Gambia) and mesoendemic (Tanzania and Niger) settings, improving the generalisability of results. Taken together, these studies provide strong evidence that increasing antibiotic coverage targets above the $80 \%$ WHO target does not reduce community ocular chlamydia or TF prevalence after 3 years of mass drug administration.

Previous studies have suggested the presence of a herd-like, indirect protection in mass azithromycin programmes for trachoma control. ${ }^{51015}$ In a hyperendemic region of Ethiopia, 


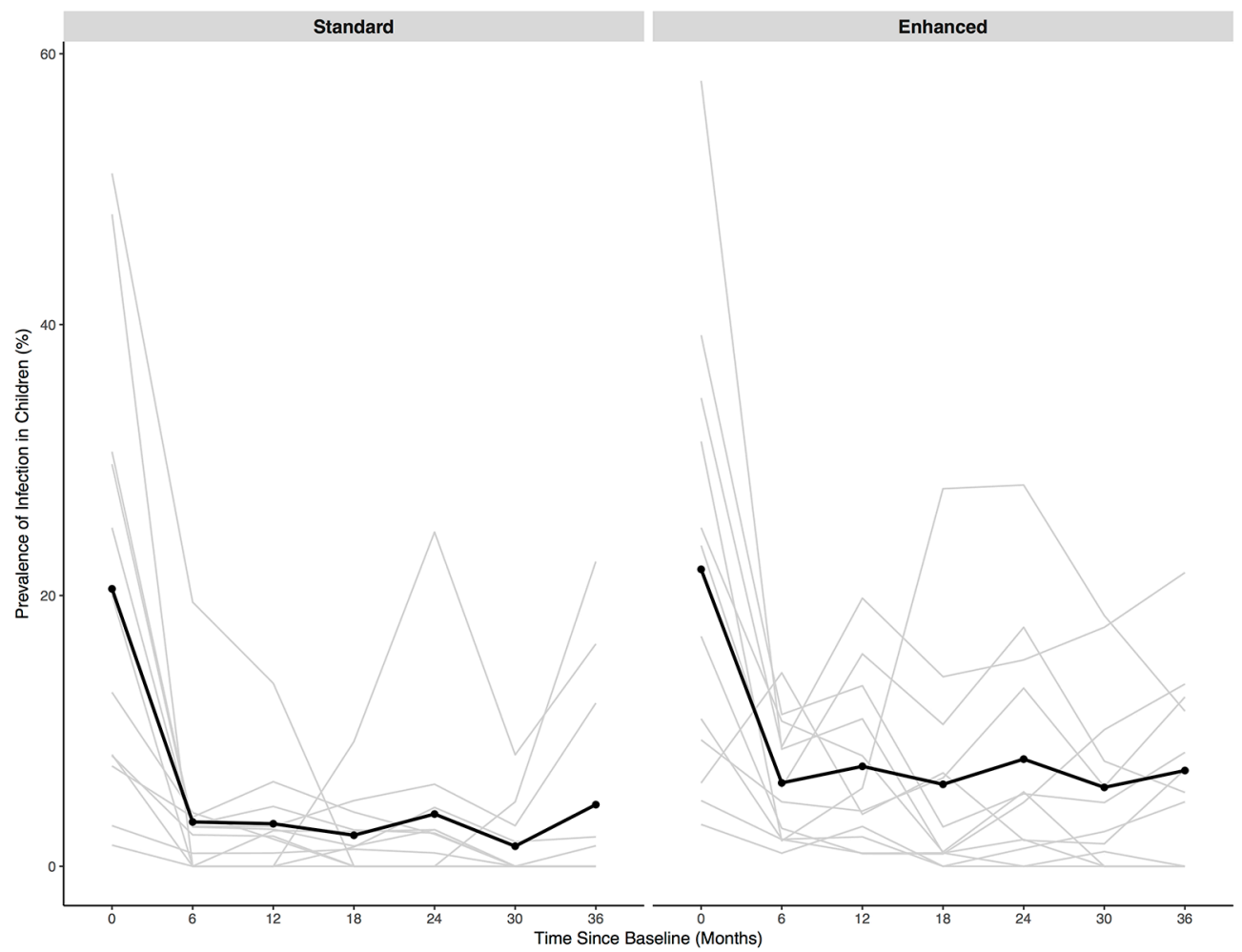

Figure 3 Prevalence of ocular chlamydia infection in children aged 0-5 years in communities randomised to standard or enhanced coverage. All communities received annual mass azithromycin treatment in all age groups. Each of the 24 communities was monitored biannually (grey curves) and the mean was calculated for all communities (black curve).

untreated children in communities receiving mass azithromycin distribution according to WHO guidelines had significantly lower odds of ocular chlamydia infection compared with children in untreated communities. ${ }^{15}$ In Niger, targeted treatment of children led to a decrease in ocular chlamydia prevalence among untreated adults. ${ }^{10}$ The lack of difference in ocular chlamydia

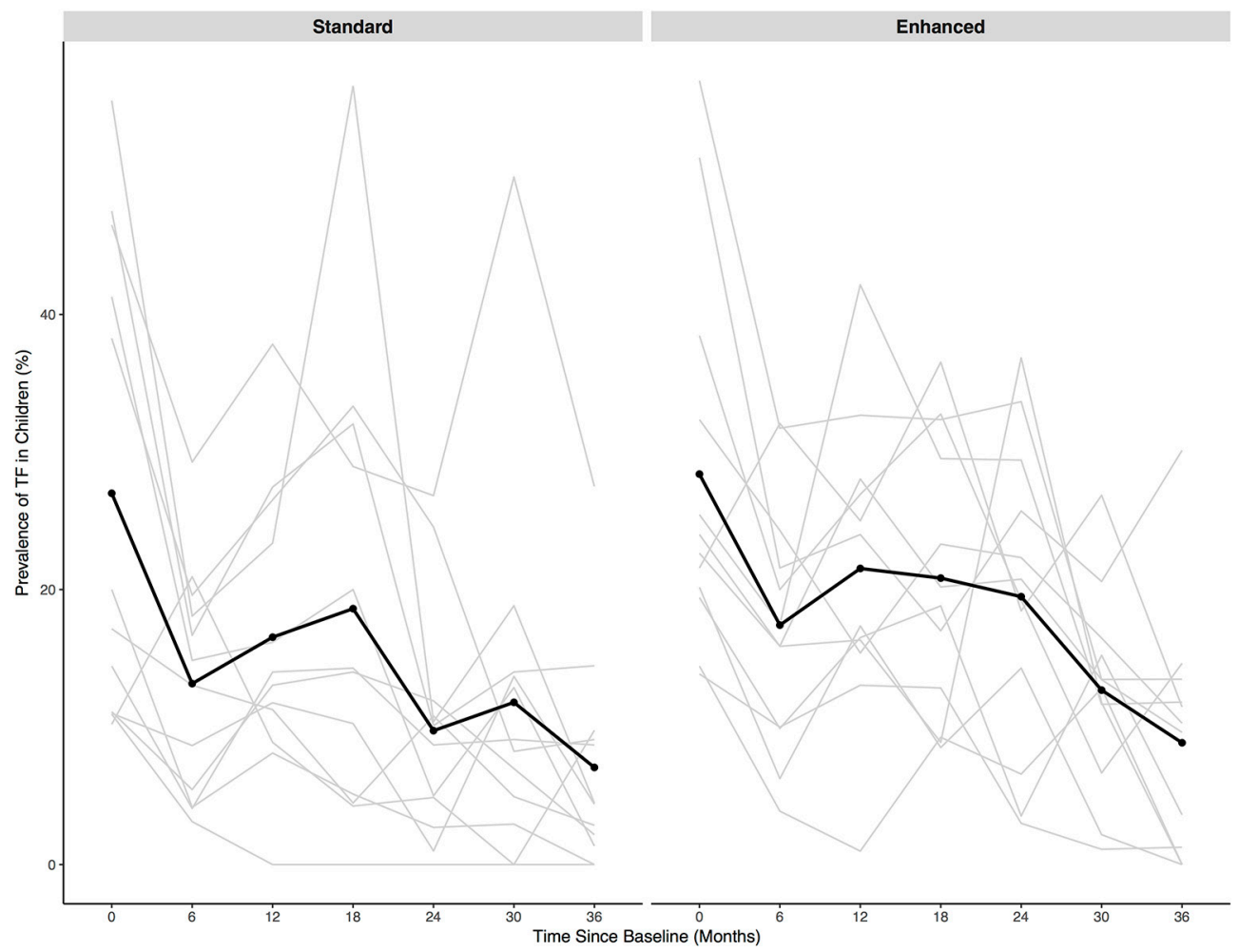

Figure 4 Prevalence of trachomatous inflammation-follicular (TF) in children aged 0-5 years in communities randomised to standard or enhanced coverage. All communities received annual mass azithromycin treatment in all age groups. Each of the 24 communities was monitored biannually (grey curves) and the mean was calculated for all communities (black curve). 
infection seen in the present study may be due to herd protection. It is possible that the $80 \%$ coverage target is sufficient to achieve adequate herd protection among untreated individuals compared with higher coverage targets, resulting in no additional benefit of higher coverage targets. It is also possible that individuals who were untreated during one round of mass azithromycin distribution received treatment during a subsequent round, and achieving $80 \%$ coverage was sufficient to effectively treat all individuals in the community at least once. Given that a single dose of azithromycin has been shown to dramatically reduce the prevalence of ocular chlamydia infection in communities, ${ }^{16}$ over a 3 -year period $80 \%$ coverage may be sufficient to substantially reduce ocular chlamydia prevalence. Finally, previous evidence from Niger has shown that children who are harder to reach were less likely to have ocular chlamydia infection. ${ }^{17}$ It is possible that the majority of children with infection are reached in a single day with the standard WHO coverage target, and children treated on additional days who are harder to reach are less likely to have infection, resulting in no discernable difference at 36 months.

In both the present study and a study from the same consortium in Tanzania, ${ }^{14}$ mean ocular chlamydia infection prevalence after three annual mass azithromycin distributions was non-significantly higher in the enhanced coverage arms. In Tanzania, the absolute difference in mean adjusted prevalence was $1.4 \%$ higher in the enhanced arm compared with the standard arm, and in Niger (present study) it was $2.2 \%$ higher in the enhanced arm compared with the standard arm. Although infection levels were very low in The Gambia after a single round of mass azithromycin distribution $(0.5 \%)$, there was slightly higher prevalence in the enhanced compared with the standard arm. ${ }^{9}$ The reason for these findings is unclear. They may be due to chance, although the finding of higher infection in the enhanced arm was consistent across all three settings. It is possible that greater selection pressure from higher coverage of azithromycin results in more azithromycin resistance in C. trachomatis in the community, although no evidence of azithromycin resistance in ocular chlamydia samples following mass azithromycin distribution was noted in Tanzania. ${ }^{18}$ Furthermore, early antibiotic use in chlamydia infection has been shown in animal models to reduce host protective immunity. ${ }^{19}$ At the population level, this could result in paradoxically higher infection prevalence in communities receiving more frequent antibiotics if overall immunity to chlamydia is reduced.

There may be benefits to limiting the amount of antibiotic distributed within a community. ${ }^{20}$ Pneumococcal resistance prevalence in nasopharyngeal samples has been shown to be significantly higher in communities receiving more frequent azithromycin distribution than recommended by the WHO compared with communities not receiving treatment. ${ }^{21}$ Although resistance has been shown to decline following removal of antibiotic selection pressure from mass azithromycin distributions, ${ }^{22}$ lower antibiotic coverage may help minimise antibiotic selection pressure and resistance. Beyond antibiotic resistance, a lower coverage target also involves fewer resources for trachoma control programmes saving costs. The enhanced coverage arm in this trial required up to three additional days of mass azithromycin distribution, beyond the single day distribution. The results of this study indicate that investing additional resources in achieving coverage beyond $80 \%$ is likely to offer little benefit.

These results must be considered in the context of several limitations. Although similar results as seen in the present study have been previously reported in hypoendemic and mesoendemic regions, these results may not be generalisable to hyperendemic settings. Increasing coverage may have differential effects in regions with a higher burden of trachoma. Here, communities were followed for a 36-month period and received treatment for the entire period. There may be differences over a longer period of time or differences in reinfection following cessation of mass antibiotic distributions.

In this mesoendemic region of Niger, we found no benefit of expanding azithromycin coverage for annual mass azithromycin distribution strategies beyond the WHO 80\% target. These results are consistent with reports from Tanzania ${ }^{14}$ and The Gambia. ${ }^{9}$ Given similar results from the three studies, there appears to be little if any additional benefit of expanding azithromycin coverage beyond the WHO 80\% target for annual mass azithromycin distribution strategies in hypoendemic and mesoendemic settings.

Contributors AA: study design, study implementation, data collection, results interpretation, drafting and approval. BK, BN, SC, NS: study design, study implementation, data collection, revision and approval. SW, RB, BDG: obtaining funding, study design, study implementation, data collection, revision and approval. TCP, JDK: study design, study implementation, data collection, data analysis, data interpretation, revision and approval. TML, obtaining funding, study design, study implementation, data collection, data analysis, data interpretation, drafting, revision and approval. CEO, study design, study implementation, data collection, data analysis, data interpretation, drafting, revision and approval.

Competing interests None declared.

\section{Patient consent Obtained.}

Ethics approval Committee on Human Research, University of California, San Francisco; Comité d'Ethique du Niger.

Provenance and peer review Not commissioned; externally peer reviewed.

(c) Article author(s) (or their employer(s) unless otherwise stated in the text of the article) 2018. All rights reserved. No commercial use is permitted unless otherwise expressly granted.

\section{REFERENCES}

1 World Health Organization. Report of the 17th meeting of the WHO alliance for the global elimination of blinding trachoma. Geneva 2013:1-56.

2 Yohannan J, Munoz B, Mkocha H, et al. Can we stop mass drug administration prior to 3 annual rounds in communities with low prevalence of trachoma?: PRET Ziada trial results. JAMA Ophthalmol 2013;131:431-16.

3 Lakew T, House J, Hong KC, et al. Reduction and return of infectious trachoma in severely affected communities in Ethiopia. PLoS Negl Trop Dis 2009;3:e376-7.

4 Melese M, Chidambaram JD, Alemayehu W, et al. Feasibility of eliminating ocular Chlamydia trachomatis with repeat mass antibiotic treatments. JAMA 2004:292:721-5

5 House Jl, Ayele B, Porco TC, et al. Assessment of herd protection against trachoma due to repeated mass antibiotic distributions: a cluster-randomised trial. The Lancet 2009;373:1111-8.

6 Chidambaram JD, Melese M, Alemayehu W, et al. Mass antibiotic treatment and community protection in trachoma control programs. Clin Infect Dis 2004;39:e95-e97.

7 West SK, Bailey R, Munoz B, et al. A randomized trial of two coverage targets for mass treatment with azithromycin for trachoma. PLoS Negl Trop Dis 2013;7:e2415-7.

8 Stare D, Harding-Esch E, Munoz B, et al. Design and baseline data of a randomized trial to evaluate coverage and frequency of mass treatment with azithromycin: the Partnership for Rapid Elimination of Trachoma (PRET) in Tanzania and The Gambia. Ophthalmic Epidemiol 2011;18:20-9.

9 Harding-Esch EM, Sillah A, Edwards T, et al. Mass treatment with azithromycin for trachoma: when is one round enough? Results from the PRET Trial in the Gambia. PLoS Negl Trop Dis 2013;7:e2115-12.

10 Amza A, Kadri B, Nassirou B, et al. A cluster-randomized trial to assess the efficacy of targeting trachoma treatment to children. Clin Infect Dis 2016.

11 Thylefors B, Dawson CR, Jones BR, et al. A simple system for the assessment of trachoma and its complications. Bull World Health Organ 1987:65:477-83.

12 Amza A, Kadri B, Nassirou B, et al. Community risk factors for ocular Chlamydia infection in Niger: pre-treatment results from a cluster-randomized trachoma trial. PLoS Negl Trop Dis 2012;6:e1586.

13 Ray KJ, Zhou Z, Cevallos V, et al. Estimating community prevalence of ocular Chlamydia trachomatis infection using pooled polymerase chain reaction testing. Ophthalmic Epidemiol 2014;21:86-91. 
14 West SK, Bailey R, Munoz B, et al. A randomized trial of two coverage targets for mass treatment with azithromycin for trachoma. PLoS Negl Trop Dis 2013;7:e2415-7.

15 Chidambaram JD, Melese M, Alemayehu W, et al. Mass antibiotic treatment and community protection in trachoma control programs. Clin Infect Dis 2004;39:e95-e97.

16 Chidambaram JD, Alemayehu W, Melese M, et al. Effect of a single mass antibiotic distribution on the prevalence of infectious trachoma. JAMA 2006;295:1142-6.

17 Amza A, Kadri B, Nassirou B, et al. The easiest children to reach are most likely to be infected with ocular Chlamydia trachomatis in trachoma endemic areas of Niger. PLoS Negl Trop Dis 2013;7:e1983-6.

18 West SK, Moncada J, Munoz B, et al. Is there evidence for resistance of ocular Chlamydia trachomatis to azithromycin after mass treatment for trachoma control? J Infect Dis 2014;210:65-71.
19 Benoun JM, Labuda JC, McSorley SJ. Collateral Damage: detrimental effect of antibiotics on the development of protective immune memory. MBio 2016;7:e01520:16-6.

20 Fry AM, Jha HC, Lietman TM, et al. Adverse and beneficial secondary effects of mass treatment with azithromycin to eliminate blindness due to trachoma in Nepal. Clin Infect Dis 2002:35:395-402.

21 Skalet AH, Cevallos V, Ayele B, et al. Antibiotic selection pressure and macrolide resistance in nasopharyngeal Streptococcus pneumoniae: a cluster-randomized clinical trial. PLoS Med 2010;7:e1000377-9.

22 Haug S, Lakew T, Habtemariam G, et al. The decline of pneumococcal resistance after cessation of mass antibiotic distributions for trachoma. Clin Infect Dis 2010;51:571-4. 